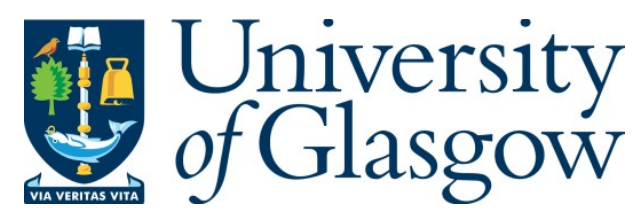

Baylan, S., and Evans, J. (2016) Development of a paradigm for studying the effects of brief Goal Management Training with Implementation Intentions. Neuropsychological Rehabilitation, (doi:10.1080/09602011.2016.1256326).

There may be differences between this version and the published version. You are advised to consult the publisher's version if you wish to cite from it.

http://eprints.gla.ac.uk/130701/

Deposited on: 9 January 2017

Enlighten - Research publications by members of the University of Glasgow http://eprints.gla.ac.uk 


\section{Development of a paradigm for studying the effects of brief Goal Management Training with Implementation Intentions}

Authors: Satu Baylan ${ }^{1}$, Jonathan Evans ${ }^{1}$

\section{Authors' Affiliation Address:}

${ }^{1}$ Institute of Health and Wellbeing, University of Glasgow, Glasgow, UK

\section{Correspondence to:}

Satu Baylan

Consultant Room 4, Zone 1

Ground Floor, Office Block,

The Queen Elizabeth University Hospital,

1345 Govan Road

Glasgow G51 4TF

email: $\underline{\text { satu.baylan@glasgow.ac.uk }}$

\footnotetext{
Abstract word count: 200 words

Manuscript word count excluding abstract, references, tables and figures: 5429 words
} 


\section{Neuropsychological Rehabilitation}

Baylan - Brief GMTii and Prospective Memory

\section{Abstract}

Cognitive rehabilitation interventions such as Goal Management Training (GMT) and Implementation Intentions have been developed with the aim of improving prospective memory (PM) in everyday life. The aim of this study was to provide 'proof of concept' for an experimental paradigm that could be used to evaluate the effects of Goal Management Implementation Intentions training (GMTii) derived from the principles of GMT and Implementation Intentions. Thirty adults were randomised to either GMTii or a control training condition. A computerised PM task that involved an ongoing task into which a PM task was embedded was completed pre-training and post-training. In addition, a novel yet similar PM task was completed post-training to assess generalisability of any effect. The two groups had similar overall performance pre-training. Post-training, the GMTii group demonstrated significantly better performance on the familiar computerised PM task showing less performance decay over time compared to the control group. The GMTii group also showed better performance on the novel task. The results demonstrated that brief GMTii significantly improved PM performance compared to control training and that computerised PM tasks were sensitive to this effect. The results suggest that this paradigm could be used to study the effects of metacognitive rehabilitation interventions.

Keywords: assessment, executive functions, conditional plans; goals; prospective memory 


\section{Neuropsychological Rehabilitation}

\section{Introduction}

Baylan - Brief GMTii and Prospective Memory

Executive functions (EF) enable individuals to solve problems, make decisions, plan for the future, execute actions at the appropriate moment and evaluate outcomes. In other words, they allow us to manage multiple overlapping goals. Prospective memory (PM) refers to the cognitive processes required for the realisation of delayed intentions critical to effective goal management in everyday life. Deficits in PM are common following acquired brain injury (ABI) and neurological disorders affecting the prefrontal cortex. Research also suggests that PM ability declines with age (Uttl, 2008). PM deficits can compromise an individual's ability to function independently as PM is needed in many everyday tasks from taking medication and cooking, to remembering to pay bills, maintaining social contacts as well as recreational activities.

Rehabilitation approaches to PM utilise passive (e.g. calendars, diaries, post-it notes and pagers) and active (e.g. NeuroPage ${ }^{\circ}$, Google calendar) external memory aids or the use of metacognitive rehabilitation interventions.

Goal Management Training (GMT) is a theoretically driven metacognitive rehabilitation intervention for executive dysfunction that has been shown to improve goal-directed behaviours following acquired brain injury (Grant et al., 2012; Levine et al., 2000; 2011), in spina bifida (Stubberud et al,. 2013) and healthy ageing (Levine et al., 2007; van Hooren et al., 2007) when delivered over multiple sessions. Studies assessing the effectiveness of brief GMT are mixed, with some supporting its usefulness (Fish et al., 2007), while others do not (Brown, 2009b). Studies integrating GMT with other intervention approaches have provided most support for its usefulness (Krasny-Pacini et al., 2014) making assessments of the 


\section{Neuropsychological Rehabilitation}

Baylan - Brief GMTii and Prospective Memory mechanism through which improvements occur challenging, given that several cognitive functions and/or psychological factors are likely to be addressed simultaneously.

Implementation Intentions (Gollwitzer, 1993; 1996) is another metacognitive strategy that has been shown to aid the realisation of a range of real-life future intentions, from losing weight (Luszczynska et al., 2007) to taking medication (Brown et al, 2009a), in addition to being able to improve performance on a multi-element task requiring PM in the laboratory (Kardiasmenos et al., 2008). It is argued that implementation intentions work by strengthening the cue-action association during the intention encoding phase (Chasteen et al., 2001). This increases the likelihood of future recall to be supported by automatic cognitive processes as opposed to generic goal intentions that require greater degree of self-generated cognitive processes.

Functional magnetic resonance imaging (fMRI) is now widely used to study brain functions with the evidence suggesting that the prefrontal cortex, in particular Brodmann area 10, plays a role in PM (see Burgess et al., 2011 for review). Tests sensitive to frontal lobe dysfunction that are typically used to assess PM ability in clinical groups tend to be complex multielement tests (Burgess et al, 2000; Levine et al, 2011; Manly et al., 2002; Wilson et al. 2005). These tests, however, are unsuitable for use with current brain imaging technology due to their complex nature. They are also likely to tap into several cognitive functions making it challenging to separate out the key cognitive processes required for PM. By contrast, simple laboratory paradigms typically used to assess PM in healthy individuals (e.g. Einstein et al., 1995) lack the complexity of clinical assessment measures and are suitable for use in a movement-constrained brain imaging environment. They typically require participants to be engaged in an ongoing task (e.g. decide whether a word is an animal) into which PM targets are embedded (e.g. make a different response when you see the word camel). We have shown 


\section{Neuropsychological Rehabilitation}

Baylan - Brief GMTii and Prospective Memory that performance on similar laboratory PM paradigms correlate with performance on multielement tasks following ABI (Baylan, 2014).Whether they are also suited for assessing the effects of cognitive training interventions is not known.

Although a few studies have examined the neural effects of cognitive training using working memory and semantic memory paradigms (Miotto et al., 2006, Osaka et al., 2012), functional changes associated with prospective memory interventions remain largely unexplored. It is important that we are able to answer questions about the mechanism of action of interventions but paradigms that involve measures that are sensitive to the cognitive construct being studied, the effects of an intervention designed to improve everyday functioning, and compatible with current brain imaging technology are lacking.

The aim of this study was to determine the suitability and sensitivity of a computerised PM paradigm in assessing the behavioural changes associated with brief training derived from Goal Management Training and Implementation Intentions (GMTii) on a familiar (previously administered) and an unfamiliar (novel) PM task. To provide proof of concept for the suitability of the paradigm to be later used for assessing functional changes in the brain associated with brief GMTii, it was expected that the GMTii group would show greater improvements in performance on the familiar computerised PM task and on the unfamiliar task, albeit to a lesser extent, following brief training compared to the control group. We were also particularly interested in whether GMTii training would reduce the extent of performance decay over the time course of the PM tasks previously observed (Baylan and Evans, 2011). 


\section{Neuropsychological Rehabilitation}

\section{Methods}

Baylan - Brief GMTii and Prospective Memory

\section{Participants}

Participants were included in the study if they were between 18 and 40 years of age, wrote with their right hand and spoke English as their first language. Exclusion criteria applied to those with a neurological illness, history of major psychiatric, psychological or substance abuse problems, those who had received treatment for psychological problems within six months prior to taking part and those who had sustained a head injury that had resulted in a loss of consciousness.

Thirty-five young individuals recruited from the University of Glasgow via advertising posters initially volunteered to take part. Two individuals were excluded due to handedness and one due to age. Of those who participated, two were excluded from the analysis for not following the task instructions in the PM condition. They both had PM target accuracy scores of 0 pre-training and post-training suggesting that they had treated the PM condition as an ongoing condition. Thus, the final sample comprised 30 individuals (15 male and 15 female), 14 of whom were in the intervention group (mean age $21.2 \pm 3.5$ years) and 16 in the control group (mean age $23.2 \pm 5.1$ years). The groups did not differ significantly with respect to their age or the amount of time they had spent in full-time education (GMTii group $15 \pm 2.3$ years; control group $16.1 \pm 2.0$ years).

\section{Measures and Materials}

Participants completed two simple event-based computerised PM tasks (Number Task and Picture Task) based on tasks from Burgess et al. (2003). The tasks were chosen because of their sensitivity to PM and their compatibility with brain imaging methods. The Number Task 


\section{Neuropsychological Rehabilitation}

Baylan - Brief GMTii and Prospective Memory was completed both pre-training and post-training. The Picture Task was completed posttraining only in order to assess the generalisability of any potential effects. Both tasks were subject paced and had two conditions: ongoing condition and prospective memory condition (identical to the ongoing condition but with an additional prospective memory instruction). Both conditions had an inter stimulus interval (ISI) that varied randomly between 150 and 350ms. Participants also performed a third task ("Seesaw Task") with a low cognitive load. The tasks were presented using E-Prime (Psychology Software Tools, Inc., USA).

In the Number Task subjects were presented with pairs of digits ranging from 1-9 (Figure 1, left). In the ongoing condition participants had to choose whether the number on the left or right hand side of the screen was greater by pressing the response button on that side. In the prospective memory condition, participants responded as in the ongoing condition but a different response (pressing a third response button on the keyboard) was required on trials where two even numbers appeared together.

In the Picture Task participants were presented with a four by six grid containing two white circles (Figure 1, middle). In the ongoing condition participants were asked to judge the orientation of the two white circles to one another by pressing the left response button if the dots were diagonal to one another and the right response button if they were horizontal. In the prospective memory condition, they were asked to continue to judge the orientation of the two white circles to one another but if both white circles were in the middle two rows of the grid, they were to press a third response button. In both the Number and Picture Tasks a PM target was presented either once (between the 3rd and 21st second) or twice (between the 3rd and 10th second and between the 14th and 21 st second) within every 30 second period at a randomly selected point. 


\section{Neuropsychological Rehabilitation}

Baylan - Brief GMTii and Prospective Memory

In the Seesaw Task, subjects were asked to tip a 'seesaw' by alternating between the left and right response buttons as fast but accurately as they could (Figure 1, right). The stimuli had an ISI varying randomly between 300 and $700 \mathrm{~ms}$.

All tasks were time limited in duration with the ongoing condition presented before the PM condition in order to prevent "contamination" of ongoing task performance (Simons, et al., 2006). Each ongoing condition lasted 2,5 minutes pre and post-training. A 30-second Seesaw Task block preceded the PM condition, which lasted three minutes pre-training and six minutes post-training. Post-training, an additional 30 second 'break' during which participants completed the Seesaw Task was presented after three minutes. The 'break' was included in order to allow us to assess whether brief GMTii could effectively reduce the occurrence of PM slips following a delay filled with an unrelated activity previously found to reduce performance on the computerised PM tasks (Baylan and Evans, 2011).

\section{Brief cognitive training interventions}

\section{Goal Management Training with Implementation Intentions}

Participants in the GMTii group were told that the purpose of the training exercise was to introduce them to strategies that can be used to improve how they use their memory. Participants were introduced to the idea of everyday memory slips through examples and were asked to rate their own prospective memory ability as excellent, good, average, poor or bad. They were then introduced to the key aspects of GMT and Implementation Intentions (Table 1) using examples from everyday life (e.g. remembering to post a letter, avoiding getting caught up when answering an exam question, passing a message onto a friend) and asked to practice the strategies by trying to apply them on three hypothetical abstract and 


\section{Neuropsychological Rehabilitation}

Baylan - Brief GMTii and Prospective Memory everyday life scenarios using a worksheet (supplementary material). The worksheet was built around concepts taken from the abbreviated GMT manuals used by Fish et al. (2007) and Brown, (2009) as well as the GMT Handbook (Levine et al., 2012; Robertson et al., 2000). The GMT instructions regarding breaking goals down into subgoal steps was replaced with the implementation intentions strategy. This was believed to be quicker to adopt with only brief training, and better suited to addressing performance decay on the computerised PM tasks which are simple in nature. Participants were also given an additional PM task (verbally), which required them to remember to circle a word describing an item of luggage (suitcase) when they came across it in the worksheet. The word was placed in the last paragraph of the handout in order have a longer delay between the task instruction and carrying out the task.

The strategies included in the GMTii intervention addressed the different stages of PM processes described by Ellis (1996) from encoding ('Stop and think' and 'if $\mathrm{x}$ happens I will do $\left.\mathrm{Y}^{\prime}\right)$ to delay (mental backboard) and evaluation (monitoring). Care was taken to ensure that no advice was given on how to apply the strategies on the computerised tasks.

\section{Control Training}

The control group received "creative thinking" training that was similar in length to GMTii but did not introduce participants to metacognitive strategies aimed at improving PM or performance on PM tasks. Participants were told that the purpose of the exercise was to get them to 'think outside the box' and they were asked to rate their creative thinking ability as excellent, good, average, poor or bad. They were then asked to work through as many of a series of lateral thinking puzzles as they could and to come up with possible solutions or explanations to the puzzles (e.g. "What can you put in a wooden box that would make it 


\section{Neuropsychological Rehabilitation}

Baylan - Brief GMTii and Prospective Memory lighter? The more of them you put in the lighter it becomes, yet the box stays empty"). A cue card was available for participants to consult, if they felt that they were not able think of a solution to a question. They were also free to move onto the next question if the cues did not help them.

\section{Procedure}

The research procedure is summarised in Figure 2.

Participants were randomly assigned to a training group after screening but before completion of the pre-training task. Randomisation was done using "single treatment per subject" online randomisation software (http://www.randomization.com) with a block length of ten. Half of the participants were allocated to Goal Management Training with Implementation Intentions (GMTii) and half to the control training. Written informed consent was obtained before taking part and participants completed a short practice block pre-training.

Testing took place in small groups over a single session lasting approximately an hour. All participants completed the Number Task prior to training. This was followed by either GMTii or control training. Both training sessions lasted 20-25 minutes. All participants completed the Number and Picture Tasks post-training. Before commencing the computerised tasks post-training, the GMTii group was told that they may find it useful to use the newly learned strategies while doing the tasks but they were not told when or how the strategies would be best used or applied on these tasks nor were the tasks used as examples during training.

This study was reviewed and approved by the University of Glasgow, Faculty of Medicine Ethics Committee and consent was completed in accordance with the Helsinki Declaration 
Baylan - Brief GMTii and Prospective Memory (http://www.wma.net/en/30publications/10policies/b3/). Participants received a payment of $£ 6$ for taking part.

\section{Analysis}

Prior to analysis, variables were screened for outliers and their normality checked using the Shapiro-Wilk test. Between-group differences were assessed using two-tailed independent ttests for normally distributed variables (appropriate adjustments for variance were made where necessary) and Mann-Whitney $U$ tests for those violating the assumption of normality.

Group $\mathrm{x}$ time interactions were assessed using repeated measures ANOVA. Where the assumptions required for ANOVA were violated or data could not be transformed to normality, change scores were analysed instead.

\section{Results}

\section{Effects of brief training on the familiar Number Task}

Performance on the computerised tasks pre-training and post-training is summarised in Table 2 and Figure 3.

Pre-training

The two groups had identical accuracies $(98.4 \%)$ in the ongoing condition $(\mathrm{U}=111.0, \mathrm{z}=-.04$, $p=.97, \mathrm{r}=-.07)$ pre-training. Both groups had similar overall accuracy in the PM condition with $96 \%$ for the control group and $96.4 \%$ for the GMTii group $(\mathrm{U}=105.0, \mathrm{Z}=-.30, p=.77$, $r=-.05)$. There was also no significant difference in the accuracy to PM targets $(U=97.0, z=-$ $.64, p=.52, \mathrm{r}=-.12)$ or ongoing trials $(\mathrm{U}=107.5, \mathrm{z}=-1.91, p=.85, \mathrm{r}=-.35)$ in the PM 


\section{Neuropsychological Rehabilitation}

Baylan - Brief GMTii and Prospective Memory condition between the two groups. Further, there were no between group differences in the reaction times in the ongoing condition $(\mathrm{U}=79.0, \mathrm{z}=-1.4, .17, \mathrm{r}=-.03), \mathrm{PM}$ condition overall $(\mathrm{T}(28)=-.806, p=.43, \mathrm{r}=0.15)$ or reaction times to PM targets $(\mathrm{t}(28)=-.64, p=.53, \mathrm{r}=.12)$ nor PM condition ongoing trials $(\mathrm{U}=82.0, \mathrm{z}=-1.25, p=.21, \mathrm{r}=-.23)$ pre-training. Given that groups did not differ significantly pre-training, it would seem reasonable to assume that any post-training differences in performance, if any, are likely to be due to the effects of the cognitive training intervention assuming that the effects of practice will be similar in both groups.

\section{Post-training}

Post-training, there were no differences in the ongoing condition accuracy in the Number Task $(\mathrm{U}=102, \mathrm{z}=-40, p=.69, \mathrm{r}=-.07)$ or reaction times $(\mathrm{U}=78, \mathrm{z}=-1.41, p=.16, \mathrm{r}=-.27$ between the two groups.

The effects of brief cognitive training whilst accounting for performance pre-training were assessed using repeated measures ANOVA or a change score from pre to post training. The change score was calculated as PM target accuracy post-training minus PM target accuracy pre-training. Positive scores indicate improvement in performance from pre- to post-training while negative scores indicate poorer performance post-training compared to pre-training. Given that both accuracy and reaction time in the ongoing condition were similar between groups, performance in the ongoing condition was not controlled for.

Both groups showed reduced accuracy to PM targets on the Number Task post-training compared to pre-training (Figure 3). The overall decrease in PM target accuracy was 5.7\% (SD 13.3) for the GMTii group and 12.3\% (SD 24.5) for the control group (Figure 3, left). 


\section{Neuropsychological Rehabilitation}

Baylan - Brief GMTii and Prospective Memory However the pattern of decrease was not significantly greater in the control group compared with the GMTii group $(\mathrm{t}(28)=-.89, p=.38, \mathrm{r}=.17)$.

Performance on the training PM exercise (remembering to circle a word describing an item of luggage), was however found to be related to the change in PM target accuracy from pre to post-training $(\mathrm{t}(12)=-2.68, p=.02, \mathrm{r}=.61)$, with those remembering to circle the word showing an improvement of $13.6 \%$ compared to a decrease of $2.1 \%$ for those who forgot.

Repeated measures ANOVA revealed no significant differences in the pattern of change in reaction times to PM targets (Figure 3, right) from pre- to post-training between groups ( $\mathrm{F}$ (1, 28) $=.73, p=.40$, partial $\left.\eta^{2}=.025\right)$.

To test whether the effects of training would be more evident in the second block following a brief break filled with an unrelated task, the performance of the two groups was compared in blocks 1 and 2, post-training by calculating a performance decay score. A summary of mean PM target accuracies is presented in Table 3 and Figure 4, right. The performance decay score was calculated by subtracting the mean PM target accuracy in block 2 from the PM target accuracy in block 1 .

The GMTii group showed a 3.3\% (SD 19.7) increase in mean PM target accuracy from block 1 to block 2, whereas the control group showed a decrease of $15.9 \%$ from block 1 to block 2 (Figure 4, left). This difference between groups was highly significant ( $\mathrm{t}(28)=-2.6, \mathrm{p}=.01$, $\mathrm{r}=.44)$.

A closer inspection of the group $\mathrm{x}$ time interaction for reaction time (Figure 4, right) suggested that greater accuracy in the second PM block shown by the GMTii may have been at a cost of increase in reaction times $\left(\mathrm{F}=(1,28)=4.72, p=.038\right.$, partial $\left.\mathrm{y}^{2}=.144\right)$. 


\section{Neuropsychological Rehabilitation}

\section{Effects of training on the Novel Picture Task}

Baylan - Brief GMTii and Prospective Memory

Performance on the Picture task is summarised in Table 4.

There were no differences in the ongoing condition accuracy $(\mathrm{U}=95.0, \mathrm{z}=-.73, p=.47 \mathrm{r}=-$ .13 ) or reaction times $\mathrm{t}(28)=-.53, p=.60, \mathrm{r}=.1)$ between groups in the novel Picture task post-training. However, the GMTii group had a significantly higher PM target accuracy (73.0\%, SD 12.7) compared to the control group (57.3\%, SD 19.2) in the PM condition ( $\mathrm{t}$ $(28)=-2.6, p=.015, \mathrm{r}=.45)$. This remained significant even after controlling for accuracy to PM targets in the pre-training Number Task $(F(1,27)=5.45, p=.027)$. The significantly greater accuracy to PM targets in the GMTii group was observed despite both groups having similar reaction times in both the PM condition overall $(\mathrm{t}(28)=-.60, p=.55, \mathrm{r}=.11)$ and to PM targets $(\mathrm{t}(28)=-.23, p=.82, \mathrm{r}=.04)$.

Performance in blocks 1 and 2 of the Picture task is summarised in Table 5.

Accuracy to PM targets in block 1 did not significantly differ between groups $(\mathrm{t}(28)=-1.1, p$ $=.29, \mathrm{r}=.05$ ). PM target accuracy reduced for both groups from block 1 to block 2 (Figure 5, left), with the GMTii group showing a reduction of $5 \%$ (SD 37.6) and the control group $20.9 \%$ (SD 31.7). The control group showed significant performance decay from block 1 to block $2(\mathrm{t}(15)=2.65, p=.018, \mathrm{r}=0.45)$, whereas the GMTii did not $(\mathrm{t}(13)=.58, p=.57, \mathrm{r}$ =0.11). The GMTii group had significantly higher PM target accuracy in block $2(\mathrm{t}(28)=-$ $.268, p=.012, \mathrm{r}=0.45)$, however, the group $\mathrm{x}$ time interaction effect was non-significant $(\mathrm{F}=$ $(1,28)=1.44, p=.24$, partial $\left.\eta^{2}=.049\right)$.

No significant differences in reaction times between groups (Figure 5, right) were observed following assessment of the group $\mathrm{x}$ time interaction $\left(\mathrm{F}(1,28)=3.3, p=.08\right.$, partial $\mathrm{y}^{2}=$ 


\section{Neuropsychological Rehabilitation}

Baylan - Brief GMTii and Prospective Memory .106). Analysis of reaction times over time for each group individually showed significant reduction in mean reaction time to PM targets from block 1 to block 2 for the GMTii group ( $\mathrm{t}$ $(13)=2.7, p=.017, \mathrm{r}=0.45)$ but not for the control group $(\mathrm{t}(15)=.43, p=.68, \mathrm{r}=0.08)$.

\section{Discussion}

This study investigated the suitability and sensitivity of a computerised PM paradigm for measuring changes in performance following brief metacognitive training derived from Goal Management Training and Implementation Intentions.

Both GMT and Implementation Intentions are theoretically derived interventions designed to improve goal attainment through raising awareness of attentional lapses, reinstating cognitive control when behaviour is mismatched to the ongoing goal hierarchy (Levine et al., 2011) and automating goal directed responses (Gollwitzer, 2006).

The paradigm was not sensitive in detecting changes in overall PM performance on the familiar Number Task following brief GMTii. However, the paradigm was sensitive in measuring changes in performance decay in the PM condition. The GMTii group was found to show better overall performance on the novel PM task with no significant performance decay from block 1 to block 2 as seen in the control group.

\section{Measuring changes on familiar task}

Goal Management Training emphasises the maintenance of attention of goal states and output monitoring over time. The GMTii intervention was effective in reducing performance decay, which reflects attentional drift in performance over time. Computerised PM tasks have been shown to be susceptible to performance decay in young neurologically healthy adults when 


\section{Neuropsychological Rehabilitation}

Baylan - Brief GMTii and Prospective Memory no strategies are used (Baylan and Evans, 2011). This finding is in line with a previous finding reporting reduction in errors of omission (i.e., not responding to go items) on the Sustained Attention Response Test (SART) following GMT (Levine et al., 2011). SART errors of omission are a measure of attentional drift and loss of top-down control (O'Connell et al., 2009).

Reduction in performance decay on the familiar Number Task suggests that the GMTii group may have benefited from the use of mental blackboard and STOP strategies of GMT and engaged in greater goal monitoring given that no cost in accuracy to the ongoing trials was seen in the PM condition. The GMTii group showed significant increases in reaction times from the first to the second PM block, compared to the control group, further supporting the application of these strategies. The observed increase in reaction times concurs with previous findings with significantly longer speed of completion having been reported on the proofreading and room layout tasks on the Simulated Real-Life Tasks (SRLTs) following GMT in healthy older adults (Levine et al., 2000). This slowing down is believed to be caused by the application of GMT principles rather than general slowing per se.

Task performance is often expected to improve with practice. Improvement in PM target accuracy on the computerised PM tasks without a break has been reported following task repetition (Burgess et al., 2003). The present finding does not support this account when performance in the PM condition is briefly interrupted with another task. In contrast, both groups showed a decline in overall performance from pre to post-training. Brief interruptions have been shown to have a negative effect on PM task performance (Walders, 2012) and previous studies assessing the effects of GMT have shown PM to be vulnerable to slips when initial high level of attention to a task has faded. Fish et al. (2007) found participants to achieve greatest success in remembering to phone an answer phone system at the correct time 


\section{Neuropsychological Rehabilitation}

Baylan - Brief GMTii and Prospective Memory in the first few days following brief GMT, which declined by midweek. A similar effect has been observed by Gracey (2012). The lack of overall effect on the Number Task in the present study may be related to the ease and fast pace of the task used, with the benefits of GMT principles only becoming evident in the second block of the task when natural performance decay occurs. It is also possible, that due to the demands of the ongoing task, participants are only able to utilise the GMTii strategies during the brief break. Alternatively or in combination with above, the short duration of the training may not have allowed all participants to be able to learn, internalise and subsequently recognise situations in which to apply these strategies given that those who showed good performance on the training exercise showed greater improvements in PM performance from pre to post-training compared to those who did not.

Individuals with executive dysfunction have particular difficulty in applying abstract rehabilitation strategies to other tasks without being provided with specific training in doing so (von Cramon and Matthes-von Cramon, 1994). With clinical samples, this should be addressed by using longer or better targeted training with advice when to use them. McDaniel et al. (2004) have shown that presenting a small blue dot on the corner of a computer screen as a reminder of the prospective memory intention improves performance following interruptions compared to when no reminder is shown but interruptions are present.

\section{Measuring changes on a novel task}

Several studies have successfully demonstrated the transfer of physical training effects to other similar untrained activities with running shown to improve cycling performance (Millet et al., 2002) and stair climbing to improve running performance for example (Loy et al., 


\section{Neuropsychological Rehabilitation}

Baylan - Brief GMTii and Prospective Memory 1993) but demonstrating the transfer and generalisability of skills following cognitive training has proved more challenging (Owen et al., 2010).

The GMTii group showed significantly greater accuracy to PM targets on the novel PM task compared to the control group. Their accuracy to PM targets was significantly higher despite having comparable reaction times with the control group in both the ongoing and PM conditions. Thus, this finding cannot be attributed to general slowing down in order to improve performance. One possible explanation is that the GMTii group may have benefited from the use of the implementation intentions strategy. The novel task is likely to require a greater degree of planning than the familiar Number Task due to a greater number of possible PM target combinations that can be encountered. The use of implementation intentions aims to strengthen the association between the PM cue (when both dots are in middle two rows) and the to-be-performed action (press $\mathrm{M}$ ) allowing the use of relatively automatic PM processes, which in turn would explain improved accuracy to PM targets and no cost to reaction times. Better performance on a non-trained laboratory task following GMT, has also recently been reported by others (Stubberud et al., 2013).

\section{Implications for further research and understanding the mechanism of change}

The findings support the view that young adults with no history of neurological illness do not spontaneously use a break to reactivate their intentions, but that it interferes with prospective remembering causing participants to disengage from the task demands (Einstein et al., 2003; McDaniel et al., 2004). Application of brief GMTii strategies can prevent performance decay on a familiar task and allow individuals to refresh their intended actions, when they are able to acquire these, and identify situations where they could be applied. How well these can be applied and adapted to real world situations that almost certainly rely on attentional, memory, 


\section{Neuropsychological Rehabilitation}

Baylan - Brief GMTii and Prospective Memory and other executive processes (Craik and Bialystok, 2006) as opposed to cognitive PM processes utilised on an isolated laboratory task needs to be investigated further.

Repetition of the PM task was not found to improve performance in the control group, which is often seen in laboratory tasks and neuropsychological assessment tools. This, together with no ceiling effects being present in either the Number or Picture Task, supports the usefulness of the computerised tasks in assessing changes in performance over time. The tasks appear sensitive to the effects of brief GMTii given that improvements in performance were only seen in the PM but not the ongoing condition. This opens up the possibility of using them to study functional changes in brain activation associated with brief GMTii, although it must be noted that some modifications will be necessary to optimise the design should fMRI be used instead of positron emission topography (PET).

Taken together, the findings suggest that performance decay may be a good indicator of how well participants are able to maintain intentions over time when other unrelated tasks are also required to be carried out as often is the case in everyday life.

Following brief GMTii, reduction in performance decay was seen in the familiar task, with a lesser reduction on the novel task. This raises a question of whether the brief training was specific enough to allow extensive transfer of application of strategy to take place. Participants were not explicitly advised when to use the strategies or prompted to think about how these could be applied on the novel task, particularly with regards to breaks. Although participants learned and practiced the strategies using a worksheet, the only self-initiated practice they were given was to remember to circle a word describing an item of luggage towards the end. Future studies should consider obtaining feedback on participants' strategy use post-training. 


\section{Neuropsychological Rehabilitation}

Baylan - Brief GMTii and Prospective Memory Although this study did not assess the generalisability of any effects to real life tasks, the evidence supporting this in the executive function domain remains particularly limited. Previous studies suggest that task-specific training is unlikely to spontaneously generalise to other everyday tasks (Cicerone and Wood, 1987; von Cramon and Matthes-von Cramon, 1994). Allowing participants to practice identifying useful situations using a hands-on task could have potentially highlighted the usefulness of the implementation intentions strategy during initial planning, the suitability of breaks in reviewing goals and could have perhaps allowed focussing on task performance when the application of the mental blackboard strategy use is not of additional benefit. Grant et al. (2012) recently administered GMT to a group of TBI patients with executive difficulties placing greater emphasis on practicing GMT principles to hypothetical everyday activities compared to previous studies (Levine et al., 2000; von Cramon and Matthes-von Cramon, 1994). GMT was found it to improve aspects of day-to-day financial management with indication of transfer of its effects with some individuals.

Individuals experiencing difficulties with PM, their families and caregivers are likely to be interested in outcomes that generalise to real world improvements in functioning. However, developing methods that allow the neural mechanism of rehabilitation interventions to be investigated has potential to further the understanding of how they work and what functional changes occur in the brain following the application of strategies that lead to improvements in everyday functioning. This in turn has potential to further the development of clinically useful cognitive rehabilitation interventions.

Although this study did not aim to evaluate the effectiveness of the brief GMTii interventions, the improvements seen in the GMTii group are encouraging given the short duration of the intervention and lack of effect reported by some previous studies 


\section{Neuropsychological Rehabilitation}

Baylan - Brief GMTii and Prospective Memory

administering brief GMT (Carstens, 2011; Wood, 2011). Use of an active rather than a passive control condition as well as the participants themselves being blind to the purpose of the study are strengths of this study. The design could be strengthened by counterbalancing tasks or by using an assessor who is blind to group allocation. It is unlikely that the latter will have influenced the findings, given that the task instructions were delivered using a computer. Future research using brief training protocols should aim to simplify strategies by highlighting characteristics of situations that benefit from their use and try to better understand which of its ingredients are key in producing changes and how to best measure these.

\section{Conclusions}

Taken together, the findings from this study lend support for the proof of concept of the potential of computerised PM paradigms to be used in the assessment of behavioural and functional changes associated with brief GMTii. The findings also suggest that the brief intervention would benefit from being better targeted to enhance its effect and to aid transfer to similar but untrained tasks before its effectiveness can be evaluated. The methods presented here may allow us to better assess the behavioural and neural mechanisms of simple interventions aimed at improving prospective goal attainment.

\section{ACKNOWLEDGEMENTS}

This work was supported by a grant from the Dr Mortimer and Theresa Sackler Foundation (S.B).

\section{CONFLICT OF INTEREST - None}




\section{Neuropsychological Rehabilitation}

\section{REFERENCES}

Baylan - Brief GMTii and Prospective Memory

Baylan, S., \& Evans, J. J. (2011). Oops, It Slipped My Mind: Improving the Assessment of Prospective Memory Using Two-Phase Computerised Tasks. Paper presented at the International Neuropsychological Society Mid-Year Meeting/ASSBI 4th Pacific Rim Conference, Auckland, New Zealand 6-9 July 2011.

Baylan, S. M. (2014). Imaging the effects of cognitive rehabilitation interventions: developing paradigms for the assessment and rehabilitation of prospective memory. (Unpublished $\mathrm{PhD}$ thesis), University of Glasgow.

Brown, I., Sheeran, P., \& Reuber, M. (2009a). Enhancing antiepileptic drug adherence: A randomized controlled trial. Epilepsy \& Behavior, 16(4), 634-639. doi: 10.1016/j.yebeh.2009.09.014

Brown, P. (2009b). Improving planning and prospective memory in a virtual reality setting: Investigating the use of periodic auditory alerts in conjunction with goal management training on a complex virtual reality task in individuals with acquired brain injury. (Unpublished D Clin Psy thesis), University of Glasgow.

Burgess, P. W., Gonen-Yaacovi, G., \& Volle, E. (2011). Functional neuroimaging studies of prospective memory: What have we learnt so far? Neuropsychologia, 49(8), 2246-2257. doi: 10.1016/j.neuropsychologia.2011.02.014

Burgess, P. W., Scott, S. K., \& Frith, C. D. (2003). The role of the rostral frontal cortex (area 10) in prospective memory: a lateral versus medial dissociation. Neuropsychologia, 41(8), 906-918. doi: $10.1016 / \mathrm{S} 0028-3932(02) 00327-5$

Burgess, P. W., Veitch, E., Costello, A. D., \& Shallice, T. (2000). The cognitive and neuroanatomical correlates of multitasking. Neuropsychologia, 38(6), 848-863. doi: 10.1016/S0028-3932(99)00134-7 


\section{Neuropsychological Rehabilitation}

Baylan - Brief GMTii and Prospective Memory

Carstens, J. (2011). The Effects of Goal Management Training in Undergraduate Students with Problems in Attention Functioning. (Unpublished Master's thesis), University of Windsor, http://scholar.uwindsor.ca/etd/222.

Chasteen, A. L., Park, D. C., \& Schwarz, N. (2001). Implementation intentions and facilitation of prospective memory. Psychological Science, 12(6), 457-461. doi: 10.1111/1467-9280.00385

Cicerone, K. D., \& Wood, J. C. (1987). Planning Disorder after Closed Head-Injury - a Case-Study. Archives of Physical Medicine and Rehabilitation, 68(2), 111-115.

Craik, F. I. M., \& Bialystok, E. (2006). Planning and task management in older adults: Cooking breakfast. Memory \& Cognition, 34(6), 1236-1249. doi: 10.3758/Bf03193268

Einstein, G. O., McDaniel, M. A., Richardson, S. L., Guynn, M. J., Cunfer, A. R., (1995). Aging and Prospective Memory - Examining the Influences of Self-Initiated Retrieval-Processes, Journal of Experimental Psychology-Learning Memory and Cognition, 21, (4): 996-1007.

Einstein, G. O., McDaniel, M. A., Williford, C. L., Pagan, J. L., \& Dismukes, R. K. (2003). Forgetting of intentions in demanding situations is rapid. Journal of Experimental Psychology-Applied, 9(3), 147-162. doi: 10.1037/1076-898x.9.3.147

Ellis, J. (1996). Prospective memory or the realization of delayed intentions: A conceptual framework for research. In M. A. Bradimonte, G. O. Einstein \& M. A. McDaniel (Eds.), Prospective memory: Theory and applications (pp. 1-22). New Jersey: Lawrence Erlbaum.

Fish, J., Evans, J. J., Nimmo, M., Martin, E., Kersel, D., Bateman, A., . . . Manly, T. (2007). Rehabilitation of executive dysfunction following brain injury: "Content-free" cueing improves everyday prospective memory performance. Neuropsychologia, 45(6), 1318-1330. doi: 10.1016/j.neuropsychologia.2006.09.015 


\section{Neuropsychological Rehabilitation}

Baylan - Brief GMTii and Prospective Memory

Gollwitzer, P. M. (1993). Goal achievement: The role of intentions. European review of social psychology, 4(1), 141-185.

Gollwitzer, P. M. (1996). The volitional benefits of planning. In P. M. Gollwitzer \& J. A. Bargh (Eds.), The psychology of action: Linking cognition and motivation to behavior (pp. 287-312). New York: Guilford.

Gollwitzer, P. M., \& Sheeran, P. (2006). Implementation intentions and goal achievement: A metaanalysis of effects and processes. Advances in Experimental Social Psychology, Vol 38, 38, 69-119. doi: 10.1013/S0065-2601(06)38002-1

Gracey, F. (2012). A randomised controlled trial of the impact of electronically delivered content-free cueing on psychosocial functioning following brain injury. Paper presented at the Ninth World Congress on Brain Injury, International Brain Injury Association, Edinburgh, March 21-25, 2012.

Grant, M., Ponsford, J., \& Bennett, P. C. (2012). The application of Goal Management Training to aspects of financial management in individuals with traumatic brain injury. Neuropsychological Rehabilitation, 22(6), 852-873. doi: 10.1080/09602011.2012.693455

Kardiasmenos, K. S., Clawson, D. M., Wilken, J. A., \& Wallin, M. T. (2008). Prospective Memory and the Efficacy of a Memory Strategy in Multiple Sclerosis. Neuropsychology, 22(6), 746-754. doi: 10.1037/A0013211

Krasny-Pacini, A., Chevignard, M., \& Evans, J. (2014). Goal Management Training for rehabilitation of executive functions: a systematic review of effectiveness in patients with acquired brain injury. Disability and Rehabilitation, 36(2), 105-116. doi: 10.3109/09638288.2013.777807

Levine, B., Manly T., Robertson, I.H. (2012). Goal Management Training Trainer Manual, Baycrest Centre for Geriatric Care, Toronto. 


\section{Neuropsychological Rehabilitation}

Baylan - Brief GMTii and Prospective Memory

Levine, B., Robertson, I. H., Clare, L., Carter, G., Hong, J., Wilson, B. A., . . Stuss, D. T. (2000).

Rehabilitation of executive functioning: An experimental-clinical validation of Goal Management

Training. Journal of the International Neuropsychological Society, 6(3), 299-312. doi: $10.1017 / \mathrm{S} 1355617700633052$

Levine, B., Schweizer, T. A., O'Connor, C., Turner, G., Gillingham, S., Stuss, D. T., . . Robertson, I. H. (2011). Rehabilitation of executive functioning in patients with frontal lobe brain damage with goal management training. Frontiers in Human Neuroscience, 5, 1-9. doi: 10.3389/Fnhum.2011.00009

Levine, B., Stuss, D. T., Winocur, G., Binns, M. A., Fahy, L., Mandic, M., . . Robertson, I. H. (2007). Cognitive rehabilitation in the elderly: effects on strategic behavior in relation to goal management. Journal of the International Neuropsychological Society, 13(01), 143-152. doi: $10.1017 / \mathrm{S} 1355617707070178$

Loy, S. F., Holland, G. J., Mutton, D. L., Snow, J., Vincent, W. J., Hoffmann, J. J., \& Shaw, S. (1993). Effects of Stair-Climbing Vs Run Training on Treadmill and Track Running Performance. Medicine and Science in Sports and Exercise, 25(11), 1275-1278.

Luszczynska, A., Sobczyk, A., \& Abraham, C. (2007). Planning to lose weight: Randomized controlled trial of an implementation intention prompt to enhance weight reduction among overweight and obese women. Health Psychology, 26(4), 507-512. doi: 10.1037/0278-6133.26.4.507

Manly, T., Hawkins, K., Evans, J., Woldt, K., \& Robertson, I. H. (2002). Rehabilitation of executive function: facilitation of effective goal management on complex tasks using periodic auditory alerts. Neuropsychologia, 40(3), 271-281. doi: 10.1016/S0028-3932(01)00094-X

McDaniel, M. A., Einstein, G. O., Graham, T., \& Rall, E. (2004). Delaying execution of intentions: Overcoming the costs of interruptions. Applied Cognitive Psychology, 18(5), 533-547. doi: 10.1002/Acp. 1002 


\section{Neuropsychological Rehabilitation}

Baylan - Brief GMTii and Prospective Memory

Millet, G. P., Candau, R. B., Barbier, B., Busso, T., Rouillon, J. D., \& Chatard, J. C. (2002). Modelling the transfers of training effects on performance in elite triathletes. International Journal of Sports Medicine, 23(1), 55-63. doi: 10.1055/S-2002-19276

Miotto, E. C., Savage, C. R., Evans, J. J., Wilson, B. A., Martins, M. G. M., Iaki, S., \& Amaro, E. (2006). Bilateral activation of the prefrontal cortex after strategic semantic cognitive training. Human Brain Mapping, 27(4), 288-295. doi: 10.1002/Hbm.20184

O'Connell, R. G., Dockree, P. M., Bellgrove, M. A., Turin, A., Ward, S., Foxe, J. J., \& Robertson, I. H. (2009). Two Types of Action Error: Electrophysiological Evidence for Separable Inhibitory and Sustained Attention Neural Mechanisms Producing Error on Go/No-go Tasks. Journal of Cognitive Neuroscience, 21(1), 93-104. doi: 10.1162/jocn.2009.21008

Osaka, M., Otsuka, Y., \& Osaka, N. (2012). Verbal to visual code switching improves working memory in older adults: an fMRI study. Frontiers in Human Neuroscience, 6 . doi: 10.3389/Fnhum.2012.00024

Owen, A. M., Hampshire, A., Grahn, J. A., Stenton, R., Dajani, S., Burns, A. S., . . Ballard, C. G. (2010). Putting brain training to the test. Nature, 465(7299), 775-U776. doi: 10.1038/Nature09042

Robertson, I., Levine, B., \& Manly, T. (2000). Goal Management Training Manual. Unpublished Manuscript.

Simons, J.S., Scholvinck, M.L., Gilbert, S.J., Frith, C.D., Burgess, P. W. (2006). Differential components of prospective memory? Evidence from fMRI, Neuropsychologia, 44, (8): 1388-1397. doi:10.1016/j.neuropsychologia.2006.01.005

Stubberud, J., Langenbahn, D., Levine, B., Stanghelle, J., \& Schanke, A. K. (2013). Goal Management Training of Executive Functions in Patients with Spina Bifida: A Randomized Controlled Trial. Journal of the International Neuropsychological Society, 19(6), 672-685. doi: $10.1017 / \mathrm{S} 1355617713000209$ 


\section{Neuropsychological Rehabilitation}

Baylan - Brief GMTii and Prospective Memory Uttl, B. (2008). Transparent Meta-Analysis of Prospective Memory and Aging, PLoS ONE, 3, (2): e1568. doi: 10.1371/journal.pone.0001568

van Hooren, S. A. H., Valentijn, S. A. M., Bosma, H., Ponds, R. W. H. M., van Boxtel, M. P. J., Levine, B., . . Jolles, J. (2007). Effect of a structured course involving goal management training in older adults: A randomised controlled trial. Patient Education and Counseling, 65(2), 205-213. doi: 10.1016/j.pec.2006.07.010

Von Cramon, D. Y., \& Matthes-von Cramon, G. (1994). Back to work with a chronic dysexecutive syndrome? (A case report). Neuropsychological Rehabilitation, 4(4), 399-417. doi: $10.1080 / 09602019408401608$

Walders, K. (2012). The effect of interruptions on prospective memory in the emergency department. (Unpublished $\mathrm{PhD}$ thesis), Rochester Institute of Technology.

Wilson, B. A., Evans, J. J., Emslie, H., Foley, J., Shiel, A., Watson, P., . . Groot, Y. (2005). Cambridge Prospective Memory Test (CAMPROMPT). London: Harcourt Assessment.

Wood, A. (2011). Rehabilitation of executive function deficits following acquired brain injury: a randomised controlled trial using Goal Management Training and Implementation Intentions to improve prospective memory. (Unpublished D Clin Psy thesis), University of Glasgow. 
TABLES

Baylan - Brief GMTii and Prospective Memory

Table 1: Brief GMTii strategies

\begin{tabular}{lll}
\hline Strategy & Purpose & $\begin{array}{l}\text { Instructions of when to use to } \\
\text { participants }\end{array}$ \\
\hline
\end{tabular}

1. Stop and Think

2. When X happens do

4. Monitoring
To draw attention to the task on hand and aid in thinking through (planning) the steps/goals of the task without being distracted by other

things

\section{To strengthen the link between what} needs to be done and when

To keep a check on things needing done later. (The use of mental blackboard was believed to minimize performance decay).

To assess whether one is doing what supposed to and to avoid information from disappearing from the mental blackboard due to limited capacity
No advise given when best to use Any time after you have created an intention; remember that there is limited amount of space on the blackboard

Check your mental blackboard regularly taking a break from what you are doing

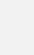




\section{Neuropsychological Rehabilitation}

Baylan - Brief GMTii and Prospective Memory

Table 2: Performance on the Number Task pre-training and post-training

\begin{tabular}{|c|c|c|c|c|}
\hline \multirow[t]{2}{*}{ Condition } & \multicolumn{2}{|c|}{ GMTii Group $(n=14)$} & \multicolumn{2}{|c|}{ Control Group $(n=16)$} \\
\hline & Pre-Training & Post-Training & Pre-Training & Post-Training \\
\hline \multicolumn{5}{|l|}{ Accuracy (\%) } \\
\hline Ongoing & $98.4 \% \pm 1.1$ & $97.6 \% \pm 1.8$ & $98.4 \% \pm 1.0$ & $97.0 \% \pm 2.5$ \\
\hline Prospective Memory & $96.4 \% \pm 2.9$ & $96.4 \% \pm 1.7$ & $96.0 \% \pm 3.2$ & $95.4 \% \pm 3.0$ \\
\hline PM trials only & $80.4 \% \pm 13.8$ & $74.6 \% \pm 15.8$ & $73.1 \% \pm 24.7$ & $60.8 \% \pm 23.1$ \\
\hline OG trials only & $97.5 \% \pm 2.6$ & $97.6 \% \pm 1.5$ & $97.4 \% \pm 2.3$ & $97.3 \% \pm 2.3$ \\
\hline \multicolumn{5}{|l|}{ Reaction Times (ms) } \\
\hline Ongoing & $656.5 \pm 106.0$ & $592.7 \pm 113.3$ & $618.5 \pm 162.7$ & $567.8 \pm 183.4$ \\
\hline Prospective Memory & $884.8 \pm 161.0$ & $754.1 \pm 156.2$ & $825.8 \pm 228.1$ & $691.7 \pm 203.9$ \\
\hline PM trials only & $939.2 \pm 160.7$ & $846.1 \pm 166.1$ & $894.7 \pm 211.6$ & $763.1 \pm 186.2$ \\
\hline OG trials only & $881.7 \pm 161.8$ & $749.3 \pm 156.7$ & $821.7 \pm 230.1$ & $688.4 \pm 205.8$ \\
\hline
\end{tabular}

Ongoing = overall performance in the ongoing condition, $\mathrm{PM}=$ Overall performance in the prospective memory condition, PM targets = performance to PM targets only in the prospective memory condition 


\section{Neuropsychological Rehabilitation}

Baylan - Brief GMTii and Prospective Memory

Table 3: Mean PM target accuracies and reaction times in Number Task prospective memory condition blocks 1 and 2 Post-training

\begin{tabular}{lcccc}
\hline & \multicolumn{2}{c}{ Group } \\
\cline { 2 - 5 } Condition & \multicolumn{2}{c}{ Control } & \multicolumn{2}{c}{ GMTii } \\
\cline { 2 - 5 } & \multicolumn{2}{c}{ Accuracy (\%) Reaction Time (ms) } & Accuracy (\%) & Reaction Time (ms) \\
& \multicolumn{2}{c}{ Mean \pm SD } & \multicolumn{2}{c}{ Mean \pm SD } \\
\hline N PM1 Block Accuracy & $68.8 \% \pm 22.5$ & $786.2 \pm 171.0$ & $73.1 \% \pm 20.0$ & $832.9 \pm 149.7$ \\
N PM2 Block Accuracy & $52.9 \% \pm 27.8$ & $740.0 \pm 210.8$ & $76.4 \% \pm 17.4$ & $859.4 \pm 191.8$ \\
\hline
\end{tabular}

N PM1 = Number Task performance to PM targets in the first prospective memory condition block; N PM2 = Number Task performance to PM targets in the second prospective memory condition block. 


\section{Neuropsychological Rehabilitation}

Table 4: Picture Task descriptive Statistics post-training

Baylan - Brief GMTii and Prospective Memory

\begin{tabular}{lcccc}
\hline \multirow{2}{*}{ Condition } & \multicolumn{3}{c}{ Group } \\
\cline { 2 - 5 } & \multicolumn{2}{c}{ Control (n=16) } & \multicolumn{2}{c}{ GMTii (n=14) } \\
\cline { 2 - 5 } & \multicolumn{2}{c}{ Mean \pm SD } & \multicolumn{2}{c}{ Mean \pm SD } \\
\hline Ongoing & $\frac{\text { Accuracy }}{\text { Peaction Time }}$ & $\frac{\text { Accuracy }}{633.6 \pm 87.9}$ & $96.8 \% \pm 0.03$ & Reaction Time \\
Picture Task & $95.1 \% \pm 5.1$ & & & \\
\hline Prospective Memory & & & & \\
Picture Task & $92.9 \% \pm 5.0$ & $807.9 \pm 149.6$ & $95.4 \% \pm 1.9$ & $841.4 \pm 154.2$ \\
$\quad$ PM targets only & $57.3 \% \pm 19.2$ & $913.0 \pm 240.8$ & $73.00 \% \pm 12.7$ & $931.3 \pm 179.3$ \\
\hline
\end{tabular}

PM denotes prospective memory; Reaction times are reported in milliseconds 


\section{Neuropsychological Rehabilitation}

Baylan - Brief GMTii and Prospective Memory

Table 5: Mean PM target accuracies and reaction times in Picture Task prospective memory condition blocks 1 and 2 post-training

\begin{tabular}{|c|c|c|c|c|}
\hline \multirow{4}{*}{ Condition } & \multicolumn{4}{|c|}{ Group } \\
\hline & \multicolumn{2}{|c|}{ Control } & \multicolumn{2}{|c|}{ GMTii } \\
\hline & Accuracy (\%) & Reaction Time (ms) & Accuracy (\%) & Reaction Time (ms) \\
\hline & \multicolumn{2}{|c|}{ Mean \pm SD } & \multicolumn{2}{|c|}{ Mean \pm SD } \\
\hline P PM 1 Block & $66.7 \% \pm 27.7$ & $951.1 \pm 268.2$ & $76.8 \% \pm 23.0$ & $1036.1 \pm 275.3$ \\
\hline P PM 2 Block & $45.8 \% \pm 27.3$ & $929.8 \pm 215.0$ & $71.0 \% \pm 23.7$ & $875.4 \pm 203.8$ \\
\hline
\end{tabular}

P PM1 = Picture Task performance to PM targets in the first prospective memory condition block; PM2 = Picture Task performance to PM targets in the second prospective memory condition block. 


\section{FIGURE LEGENDS}

Baylan - Brief GMTii and Prospective Memory

Figure 1: Computerised task stimuli. The figure on left shows the Number Task stimuli, the figure in the middle the Picture Task stimuli and the figure on the right the Seesaw Task stimulus.

Figure 2: Research procedure flowchart

Figure 3: Mean Number Task accuracy (left) and reaction times (right) to PM targets pretraining and post-training. Error bars represent $95 \%$ confidence intervals.

Figure 4: Mean Number Task accuracy (left) and reaction times (right) to PM targets before and after a brief break filled with unrelated task post-training. Error bars represent $95 \%$ confidence intervals.

Figure 5: Mean Picture Task accuracy (left) and reaction times (right) to PM targets before and after a brief break filled with unrelated task post-training. Error bars represent $95 \%$ confidence intervals. 


\section{FIGURES}
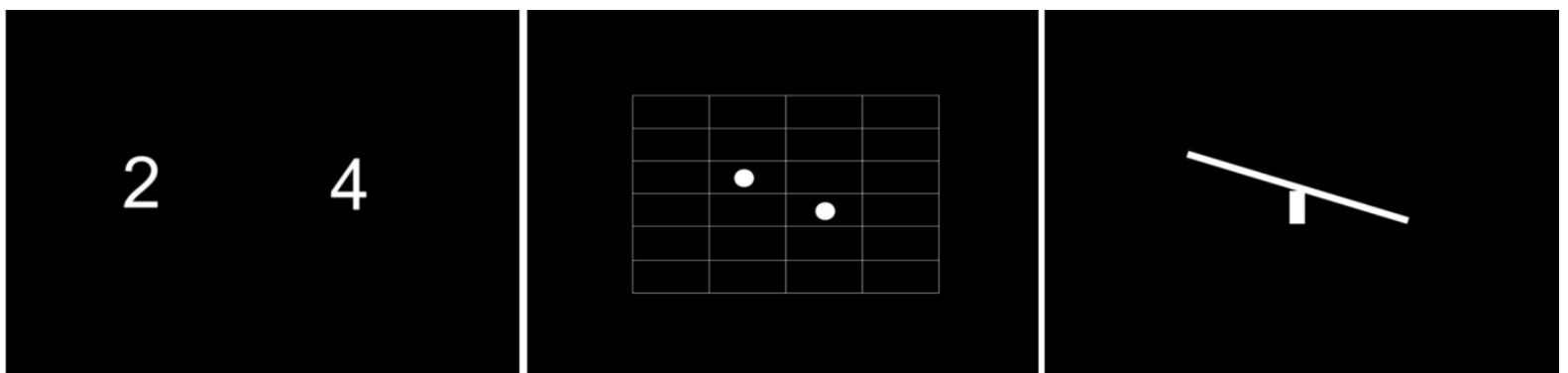

Figure 1 


\section{Neuropsychological Rehabilitation}

Baylan - Brief GMTii and Prospective Memory

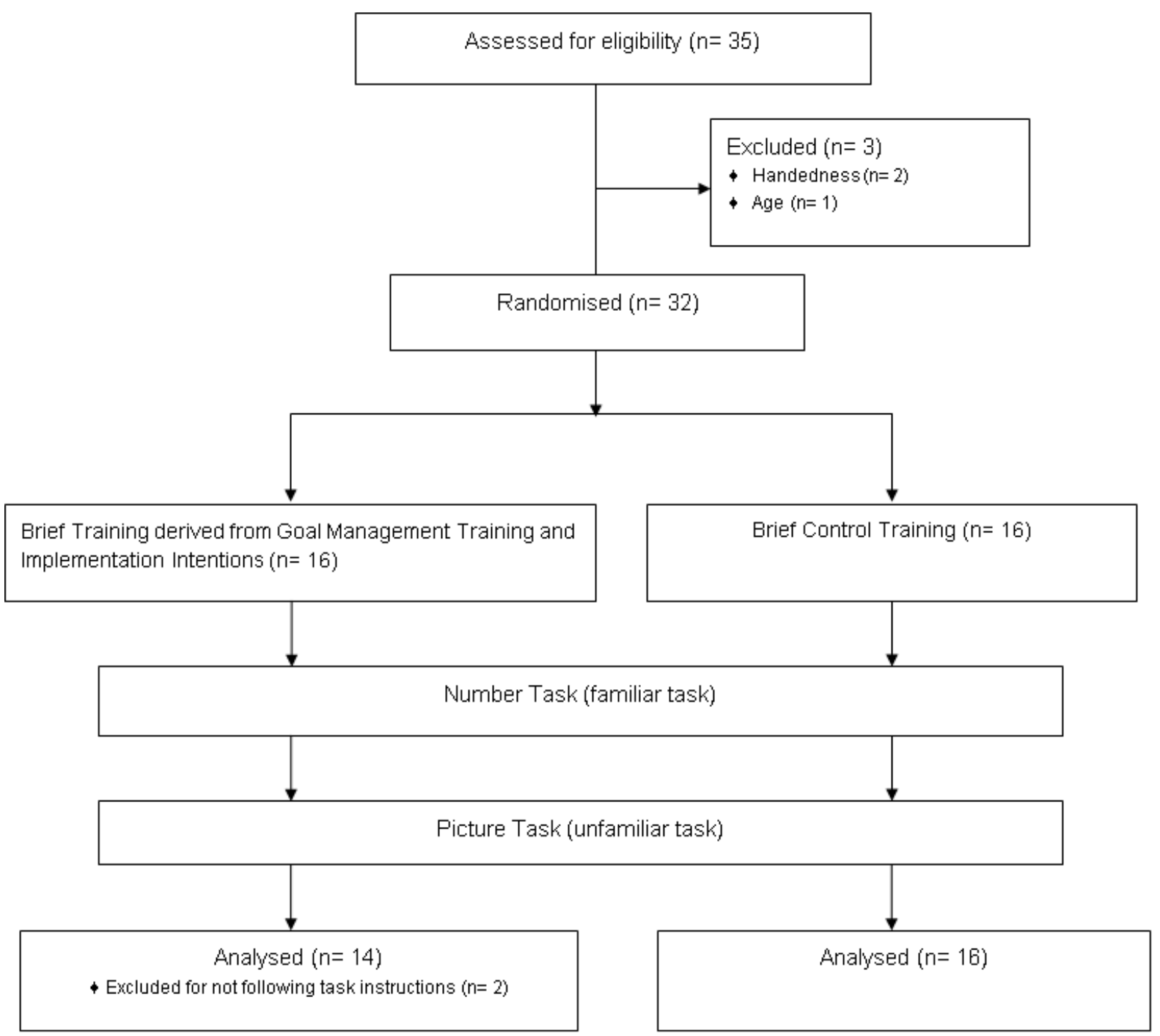

Figure 2 


\section{Neuropsychological Rehabilitation}

Baylan - Brief GMTii and Prospective Memory

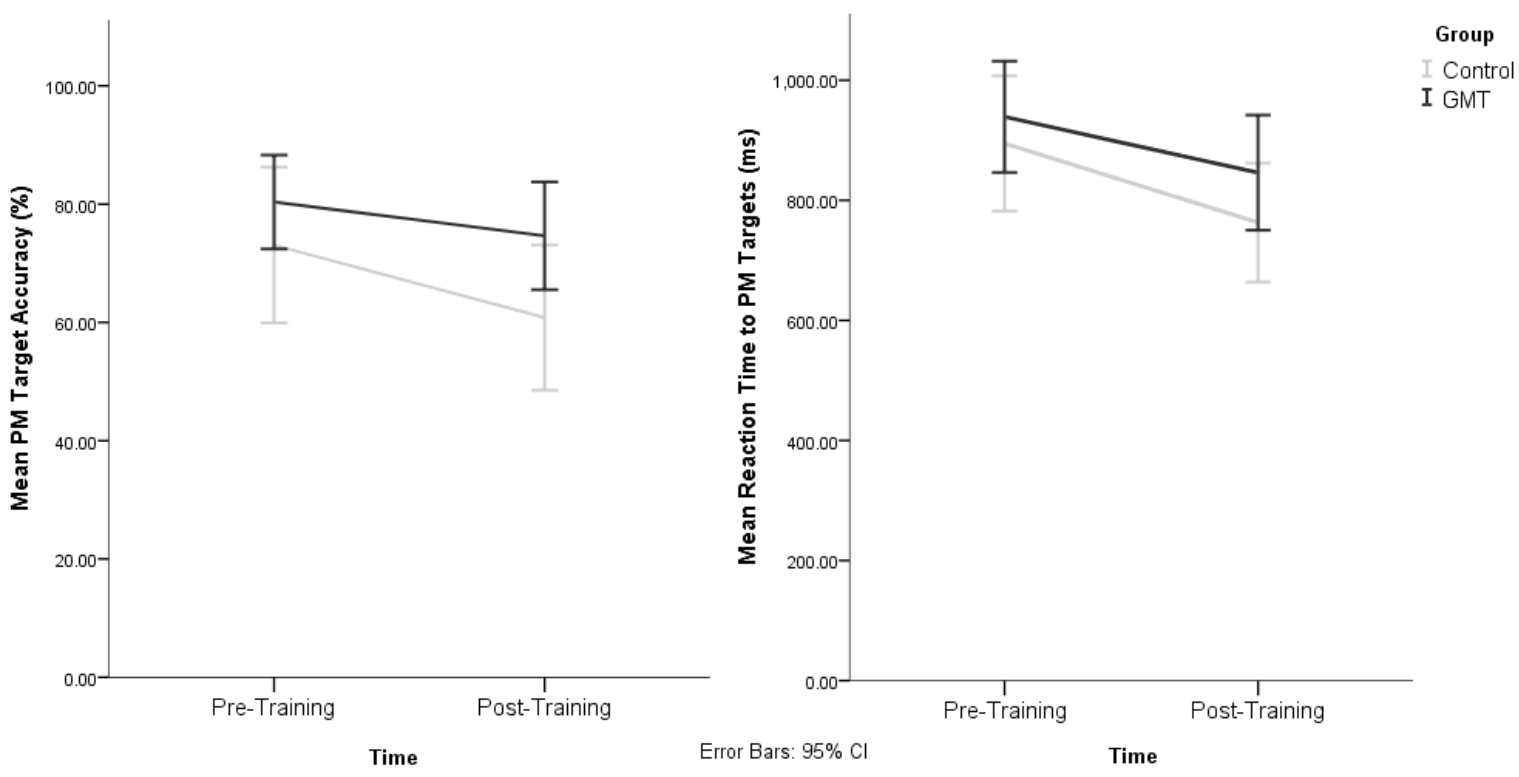

Figure 3 


\section{Neuropsychological Rehabilitation}

Baylan - Brief GMTii and Prospective Memory

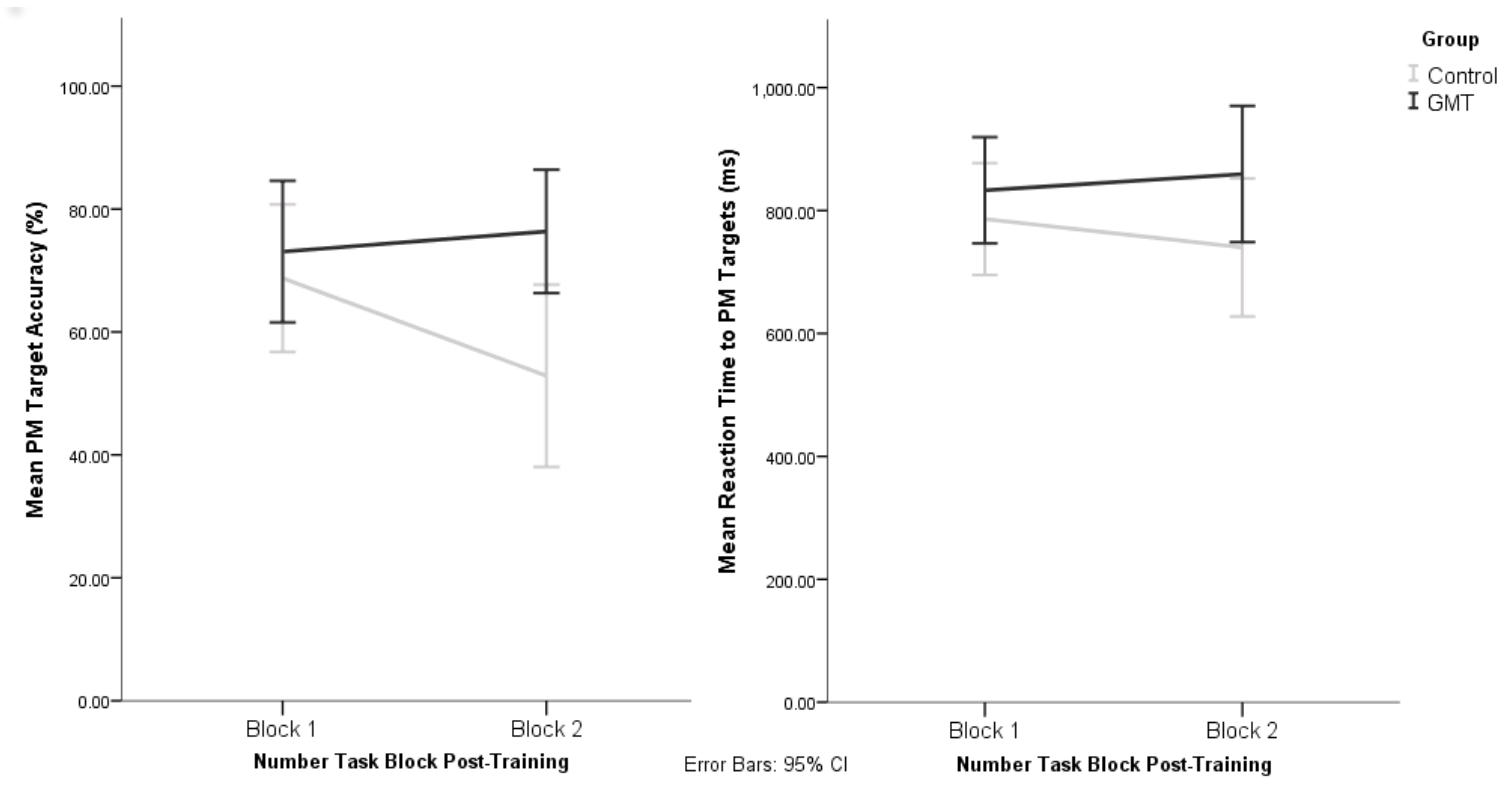

Figure 4 


\section{Neuropsychological Rehabilitation}

Baylan - Brief GMTii and Prospective Memory

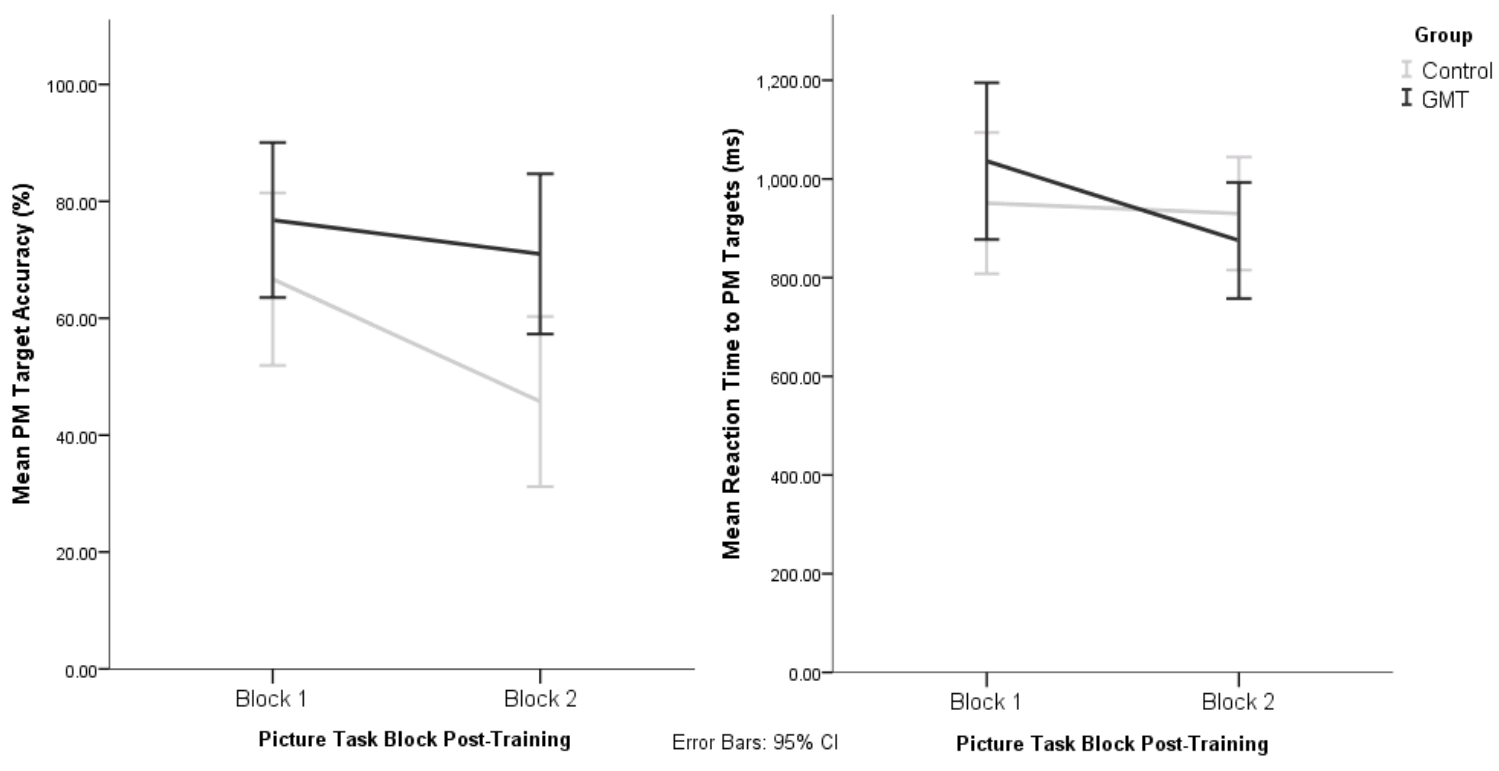

Figure 5 